Mohamed Tanta, José Cunha, Vítor Monteiro, António P. Martins, Adriano S. Carvalho and João L. Afonso

"A Novel Hardware Protection Scheme for a Modular Multilevel Converter Half-Bridge Submodule"

IECON 2019 - 45th Annual Conference of the IEEE Industrial Electronics Society, Lisbon, Portugal

To cite:

M. Tanta, J. Cunha, V. Monteiro, A. P. Martins, A. S. Carvalho and J. L. Afonso, "A Novel Hardware Protection Scheme for a Modular Multilevel Converter Half-Bridge Submodule," IECON 2019 - 45th Annual Conference of the IEEE Industrial Electronics Society, Lisbon, Portugal, 2019, pp. 6043-6048, doi: 10.1109/IECON.2019.8927361. 


\title{
A Novel Hardware Protection Scheme for a Modular Multilevel Converter Half-Bridge Submodule
}

\author{
Mohamed Tanta ${ }^{1}$, José Cunha ${ }^{1}$, Vítor Monteiro ${ }^{1}$, António P. Martins ${ }^{2}$, Adriano S. Carvalho ${ }^{2}$ and João L. Afonso ${ }^{1}$ \\ ${ }^{1}$ Centro ALGORITMI - University of Minho, Guimarães - Portugal \\ ${ }^{2}$ SYSTEC Research Center - University of Porto, Porto - Portugal \\ ${ }^{1}$ \{mohamed.tanta | jose.cunha | vitor.monteiro | joao.l.afonso\}@algoritmi.uminho.pt, ${ }^{2}\{$ ajm | asc $\} @$ fe.up.pt
}

\begin{abstract}
The Modular Multilevel Converter (MMC) has become progressively an attractive solution for the medium-power and the high-power applications, due to its modularity, flexibility of redundancy or scalability, high efficiency and low production of harmonic contents. However, in order to have a reliable and robust MMC system, different concerns should be observed in terms of the converter control complexity and the required effective protection system. In this context, this paper presents a novel hardware protection topology for the MMC half-bridge submodule. The introduced scheme is based on new sensors technology that can be assembled near the power hardware, resulting in less perturbation and a very low signal delay. This allows the protection system to respond quickly to fault situations. The proposed protection scheme of half-bridge MMC submodules is a new contribution given the steadily increasing popularity of the MMC and that just a few scientific publications treat in detail the protection mechanisms of half-bridge submodules for MMC. The experimental results confirm the effectiveness of the proposed scheme after creating the fault overvoltage and overcurrent conditions on one MMC half-bridge submodule.
\end{abstract}

Keywords - Half-bridge submodule, Modular Multilevel Converter, Overvoltage Protection, Overcurrent Protection.

\section{INTRODUCTION}

Nowadays, the multilevel converters are appearing as a new class of power converter options. Some of them have already been marketed for the commercial use, others are still in the research and development phase [1]. The Modular Multilevel Converter (MMC) is a promising solution for high and medium-power applications. This converter was firstly used in 2010 for the high-voltage direct-current (HVDC) power transfer application, in Trans Bay Cable project, San Francisco, USA [2]. Since that successful experience, the MMC has drawn more attention from the researchers, as an attempt to involve the MMC with different high and medium-power applications, especially for the Flexible AC Transmission Systems (FACTS). In this context, the investigation and installation of the Static synchronous compensator (STATCOM) based on MMC have been described in [3]. A study of a shunt active power conditioner (SAPC) based on MMC has been presented in [4] as a medium-power application. One of the other possible employments of the MMC can be in the railway electric traction systems, in which the static frequency converter (SFC) based on MMC as a medium-voltage transformerless converter can be used to directly supply the catenary overhead line as presented in [5]. Moreover, the application of a Rail Power Conditioner (RPC) system based on MMC technology for power quality improvement in railway electrification has been presented in [6], [7]. An integrated energy storage system based on MMC to interface the voltage batteries to the public grid was demonstrated in [8]. Electric Vehicles driving and fast charging converter based on MMC has been described in [9].

In such cases, reliability and the stable performance of the MMC are one of the most important demands, including the protection of any dangerous consequences that could appear because of the MMC malfunctioning, bearing in mind that MMC involves many power electronic devices such as the insulated gate bipolar transistors (IGBTs), diodes, etc., and each device might be a possible failure point. In this context, and since the MMC is composed by multi-cascade connected submodules, this paper pays much attention about the importance of MMC protection system. The paper also presents a novel hardware protection scheme against overvoltage and overcurrent conditions and it presents experimental results, showing the time response of the proposed protection.

In this context, this paper is organized as follows: section II presents an overview about the MMC and the importance of protection system, section III describes the proposed protection scheme for each MMC submodule, then providing a total protection for all MMC components. The results of MMC protection scheme as laboratory experimental tests are presented in section IV, to show the effectiveness of the proposed protection scheme. Finally, the main conclusions of this paper are presented in section $\mathrm{V}$.

\section{DESCRIPTION OF THE MMC AND THE IMPORTANCE OF PROTECTION}

Fig. 1(a) shows a three-phase DC/AC MMC constituted by six arms. Each arm is composed by $n$ number of submodules and an arm inductor as a coupling filter. A bigger value of $n$ signifies a higher MMC output waveforms level, a smaller harmonic distortion, additional control complexity and more robustness against submodule fault situations (the greater the value of $n$, the MMC can continue operating with fewer disturbances when one of the submodules is broken-down). The upper and the lower arms in the same phase constitute a phase unit. Each submodule, as shown in Fig. 1(b), involves top and bottom IGBT switches $\left(T_{1}, T_{2}\right)$, two antiparallel diodes $\left(D_{1}, D_{2}\right)$, and one capacitor $C_{s m}$. The total DC-link voltage of the submodules in one arm should be able to withstand the entire converter DC-link voltage $V_{d c}$. By another meaning, each MMC 
arm should have a total DC-link voltage with a value equal to the entire converter DC-link voltage $V_{d c}[10]$.

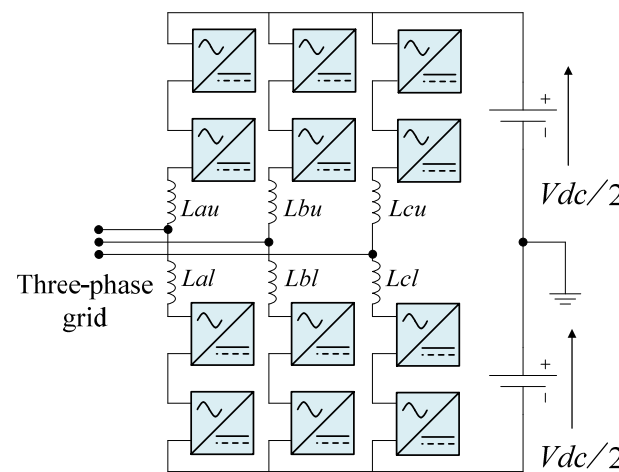

(a)
Single Submodule

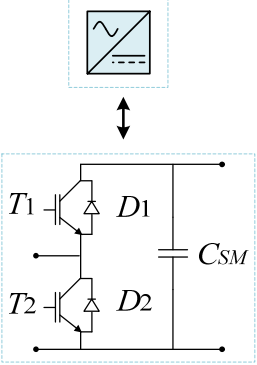

(b)
Fig. 1. Modular Multilevel Converter (MMC) topology: (a) MMC circuit diagram; (b) Single submodule diagram.

The submodule capacitor can be inserted or bypassed to produce the output AC waveform. When the submodule is required to be switched into the arm circuit, $T_{1} / D_{1}$ will be switched on and $T_{2} / D_{2}$ will be switched off. In this case, the submodule capacitor is charging or discharging according to the submodule current direction. However, the opposite scenario is when the submodule outputs a zero voltage into the arm circuit. In this case, $T_{2} / D_{2}$ will be switched on and $T_{1} / D_{1}$ will be switched off. As a result, the submodule capacitor will be always bypassed regardless the submodule current direction [11]. A deadtime band is required to avoid a short circuit fault between the switch on and the switch off states. Therefore, and in such situations, when the system is in the pre-energized state, a fault, or deadbands between the upper and the lower switches, the submodule capacitor can be either inserted or bypassed according to the submodule current direction, which flows in this case only through the diodes $D_{1}$ or $D_{2}$ [11].

There are different faults that could negatively affect the MMC operation. These faults can be sorted according to their origins: external faults caused by an external power source connected to the submodules [12] (such as the main DC-link or the three-phase grid faults as shown in Fig. 2 [13]) and the internal faults caused by the malfunctioning of one or more submodules (such as an open circuit fault in the submodule as shown in Fig. 3(a) and the deadtime fault between $T_{1}$ and $T_{2}$ as shown in Fig. 3(b)). It noteworthy to mention that the proposed protection scheme in this paper is only effective to protect from the internal MMC faults that could appear in the submodules, such as short circuit current caused by a deadtime fault, open circuit fault or short circuit fault situations resulting from the malfunctioning of one or more devices in the submodule $\left(T_{1}, T_{2}, D_{1}, D_{2}\right)$. The open circuit fault could occur as a consequence of switch blow, in which the Printed Circuit Board (PCB) traces could overheat, resulting an open circuit fault.

When an internal fault happens in the submodule, the consequences can be varied between high current path or extreme imbalance between submodules DC-voltages, in which, some submodules capacitors can be charged to exceed the capacitor nominal voltage and others can be discharged.
Once the submodule capacitor is charged with an absolute value higher than its rated voltage, the chance for a catastrophic event increase. The capacitor will overheat, and the failure could be fast with an accompanying explosion, or it might be slower with an associated reduction in the capacity, shorter lifespan and degrading the capacitor main parameters such as the equivalent series resistor and the equivalent series inductance. Therefore, overvoltage protection is required for each MMC submodule to avoid damaging effects, then avoiding the converter total collapse.

On the other hand, the overcurrent protection is extremely required in case of any switch failure in the submodule because the stored energy in the affected submodule capacitor will be released, leading to a very high-current path and accompanied by switches overheating [14]. The main aim is to suppress the fault current that exceeds the peak current value that the semiconductors (especially the antiparallel diodes $D_{1}$ and $D_{2}$ ) can withstand. Normally, the IGBT power switches contain soft antiparallel diodes that have a nominal current lower than the one of the IGBT. Therefore, the overcurrent protection must be calibrated to actuate at the nominal current values of the antiparallel diodes but not at the ones of IGBTs. On the other hand, the overcurrent protection must prevent the fault current to reach the saturation current value of the inductors between MMC legs. A large arm inductance can limit the fault current and the circulating currents between MMC arms [15]. However, using only the inductance to overcome the fault current is not enough, but it can help to dominate the fault status until the protection is activated, keeping in mind that using a big inductance for high current applications can bring a significant voltage drop, resulting in decreasing the modulation index in the linear operation of the MMC [14]. In addition, an air core inductor is recommended to avoid the saturation due to the fault current.

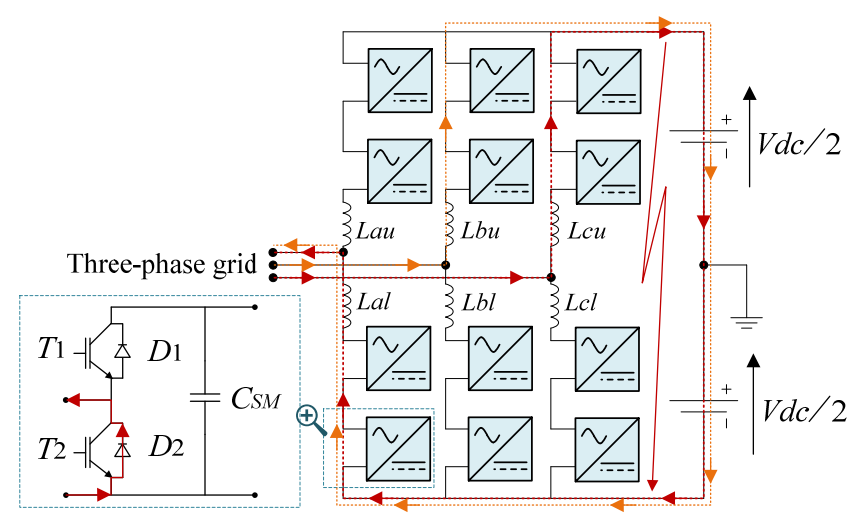

Fig. 2. MMC external origin faults.

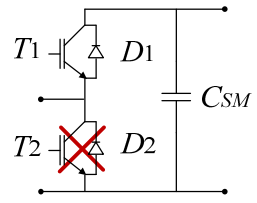

(a)

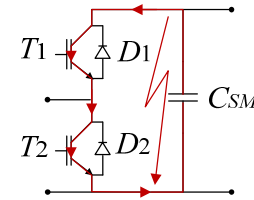

(b)
Fig. 3. MMC internal origin faults. 


\section{THE PROPOSED PROTECTION SCHEME}

As shown in Fig. 4, the proposed protection scheme is applied on each MMC submodule and it consists of two main parts: the overcurrent protection and the overvoltage protection. There are two isolated current sensors from Allegro ACS730 that measure the instantaneous current on the collector of each semiconductor (IGBT). The output of these sensors are differential to be less sensitive for any electromagnetic interference noise. The over current protection is actuated only when the actual current value across any of the IGBTs collectors $i_{a c t}$ exceeds the reference current value $i_{\text {ref }}$, as shown in the flowchart presented in Fig. 5(a). The actuating response of these sensors is very quickly to fault conditions $(0.75 \mu \mathrm{s}$ for the isolated signal amplifier si8920 and $0.2 \mu$ s for the current sensor $A C S 730$ - without applying signal filtering), which provides robust and reliable protection against short circuit faults. The flow chart diagrams in Fig. 5 is implemented with analog circuits (operation amplifiers, comparators and so on).

When a fault current is detected by any current sensors in the system, a disable signal will be triggered to bypass all the controllable semiconductors (IGBTs) in the system until an external reset action is released manually after avoiding the fault origins, such as, the deadtime fault that can cause a high short circuit current flowing through $T_{1}$ and $T_{2}$. A delay function before the reset is important to prevent the switching stress in the components. Normally, this protection is adjusted to be actuated when the current value exceeds the antiparallel diodes nominal current values, taking into consideration the saturation current of the MMC arm inductance.

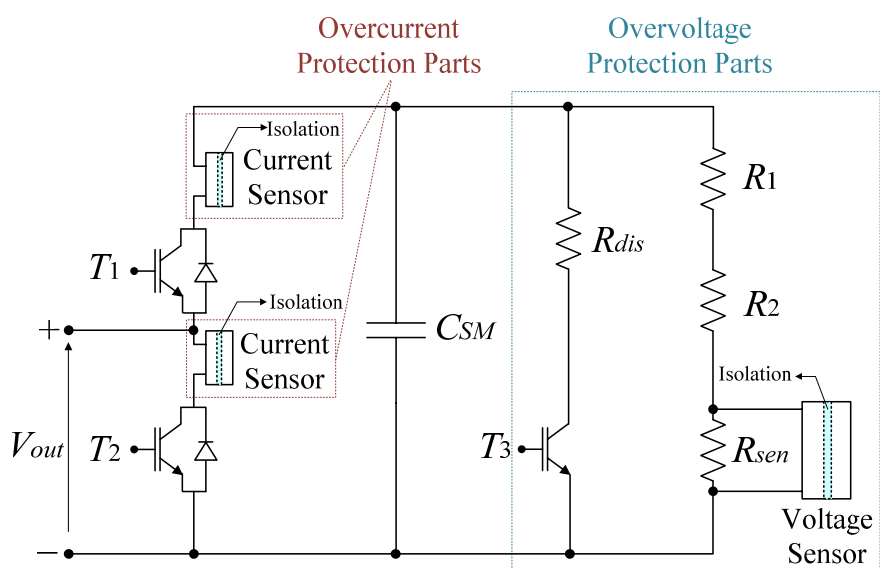

Fig. 4. Half-bridge MMC submodule and the proposed protection scheme.

The proposed overvoltage protection consists of an isolated signal amplifier Si8920 from Silicon Labs that measures the DC-voltage value across the submodule capacitor by using the measuring resistor $R_{\text {sen }}$ as shown in Fig. 4 . The voltage divider (resistors of $R_{1}, R_{2}$ and $R_{\text {sen }}$ ) is used to fit the voltage sensor nominal input value. The input and the output of this voltage sensor, besides the signal conditioning process, are differential to reduce the noise that could be resulting from the electromagnetic interference. According to the flowchart presented in Fig. 5(b), the overvoltage protection compares the actual voltage value with its high and low reference $v_{\text {refH }}$ and $v_{\text {refL }}$. The protection is actuated when the actual voltage value is equal or exceeds the reference signal. In this case, the switch $T_{3}$ will be enabled to discharge the submodule capacitor through the resistor $R_{d i s}$ until the actual voltage signal is less than the lower reference signal value $v_{\text {reft }}$. The switch T3 has no antiparallel diode since its functionality is useless at that case. Although this method of protection is not completely new. However, it is off course granted that its use is new in the context of overvoltage protection in MMC submodules.

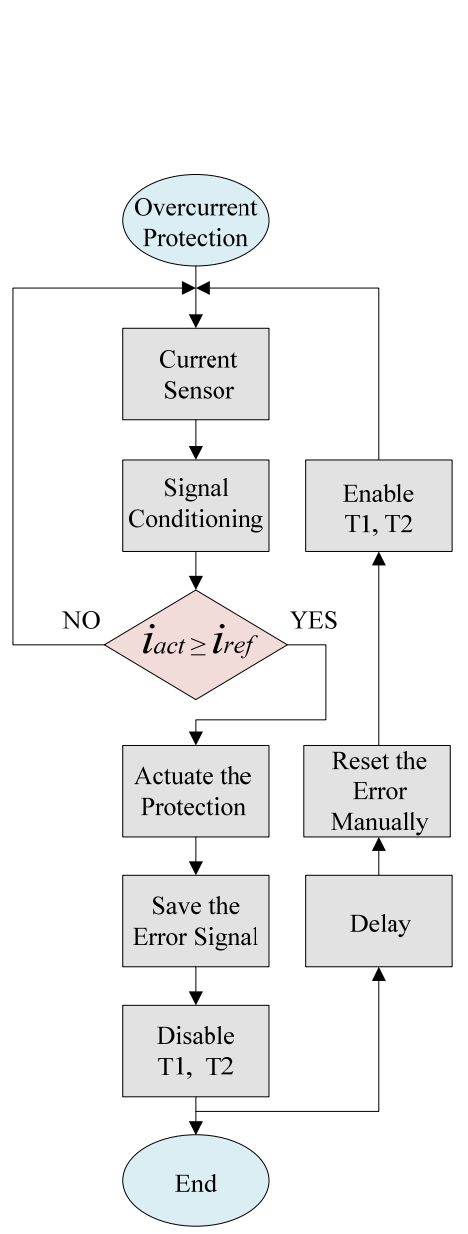

(a)

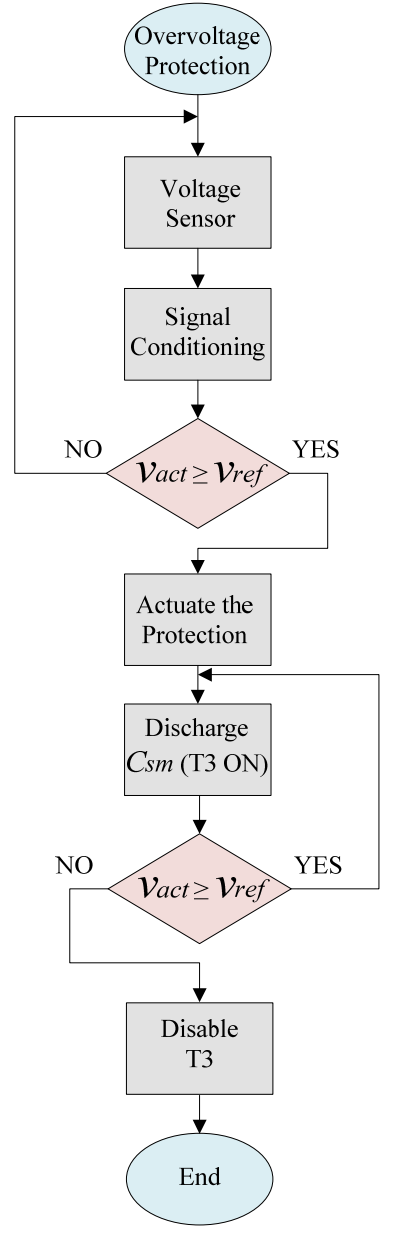

(b)
Fig. 5. Flowchart diagrams of the proposed protection scheme: (a) Overcurrent protection flowchart; (b) Overvoltage protection flowchart.

\section{EXPERIMENTAL RESULTS}

The hardware implementation of the proposed protection scheme is shown in Fig. 6, where the top side of the protection board has the main terminals for both voltage and current sensors, as well as the required components for signal conditioning process as shown in Fig. 6(a). On the other hand, the bottom side of the protection board contains the voltage and the current sensors, besides the integrated circuits (ICs) that are responsible for signal conditioning and signal comparators as shown in Fig. 6(b). There are also additional elements of resistors and capacitors to calculate the gain and to achieve signal filtering effects. 

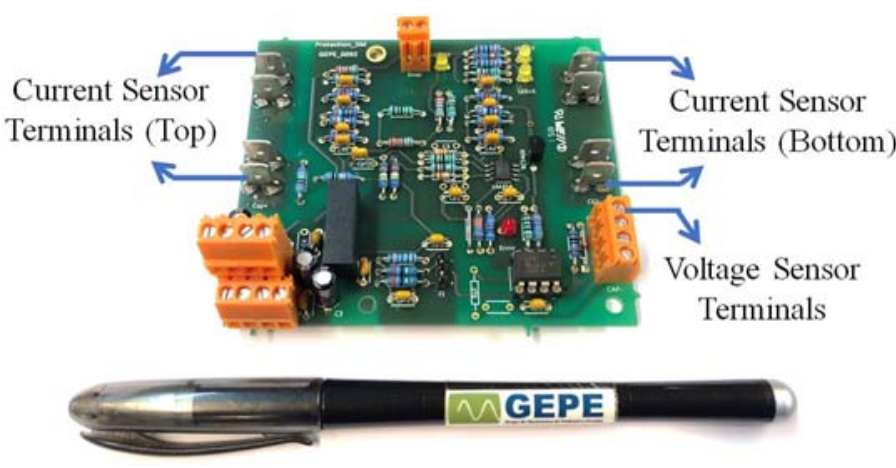

(a)
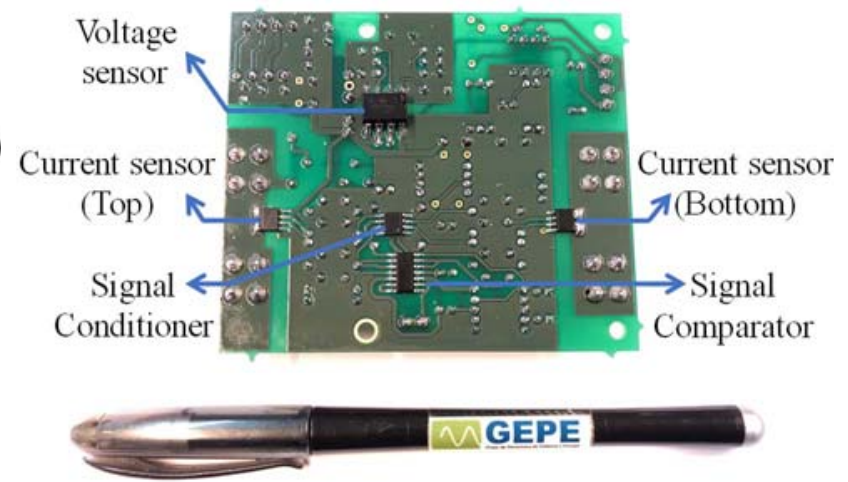

(b)

Fig. 6. Overvoltage and overcurrent protection board: (a) Protection board (top side); (b) Protection board (bottom side).

\section{A. Overcurrent Protection Test}

Testing the overcurrent protection of the MMC submodule requires to use a test workbench to verify the correctness of the current sensors under test conditions without damaging any other parts in the submodule. Therefore, in Fig. 7 is presented the schematic of the overcurrent circuit implemented in the test workbench, which consists of an adjustable auto-transformer connected to the input of a step-down transformer. The output of this transformer can stand with a high current value around $60 \mathrm{~A}$ to test the overcurrent protection. An uncontrolled rectifier bridge is connected to the output side of the step-down transformer. The electrical impedance $Z$ reflects the parasitic elements of the circuit. The isolated current sensor is connected in series with an IGBT to the rectifier bridge DC output. This series connection between the current sensor and the IGBT is used to create the correct operating conditions when a real MMC submodule is under operation. The bridge rectifier is used to keep the antiparallel diode in the reverse-biased mode and in order to have a fully controllable current.

Fig. 8 shows the experimental results of the overcurrent protection test. The protection has been adjusted to actuate when the test current exceeds an absolute value higher than $\pm 28 \mathrm{~A}$. The test current $i_{\text {test }}$ (in green) has a peak value equals to $32 \mathrm{~A}$ and the overcurrent protection signal $v_{p r}$ (in purple) is actuated when the test current exceeds the reference value $( \pm 28 \mathrm{~A})$. The differential output signal of the current sensor $v_{\text {dif }}$ (in orange) has almost the same shape of the test current signal with some extra noise. However, this noise is highly mitigated after conditioning the output signal of the current sensor $v_{\text {dif }}$ by using differential amplification. There is a very small delay (less than $1 \mu \mathrm{s}$ ) between $i_{\text {test }}$ and $v_{\text {amp }}$ signals. This delay is resulting from the current sensor and the signal conditioning delay, bearing in mind there is always a tradeoff between the signal fast response and the noise cancellation. The hysteresis amplifier as an example guarantees a cleaner output signal accompanied by a bigger delay.

Fig. 9 presents the disable response at a smaller time scale in order to assess the delay values with more precision. When the signal of $v_{p r}$ equals to $-15 \mathrm{~V}$, the $P W M$ pulses should be low in order to open the IGBT power switches. The signal of $v_{\text {disable }}$ is the one applied on the disable pin of the driver chip (Si8244 from Silicon Labs) and it climbs to reach $5 \mathrm{~V}$ when the signal $v_{p r}$ is negative. The $P W M$ pulses of the top and bottom submodule switches have a switching frequency of $40 \mathrm{kHz}$. The results show a response delay from the overcurrent protection actuation until the protection response equals to $1 \mu \mathrm{s}$. There is also an additional delay that is resulting from the detection of the overcurrent state estimated by $2 \mu \mathrm{s}$ (this time delay is not presented in Fig. 9). The overall inevitable delay (estimated by $3 \mu \mathrm{s})$ arises from several factors related to hardware propagation, such as sensor lags in the protection board (estimated by $0.2 \mu \mathrm{s}$ for the allegro ACS730), signal processing delay in the command board (estimated by $2.5 \mu \mathrm{s}$ ) and the internal disable delay of the driver chip (estimated by $0.1 \mu \mathrm{s}$ ). Another important point, in analog systems, most of the delay time response is caused by the opto-coupled devices, such as optocouplers. Therefore, and since this protection is using complementary metal oxide semiconductor (CMOS) isolation, so it is important to use the same isolation technology in the analog control integrated circuits. Consequently, optocouplers in the analog control were replaced by new devices, LED emulator input, open collector output isolators (Si8710 from Silicon labs). These devices have a faster response and a higher isolation capability than the normal optocouplers. The response delay from the overcurrent protection actuation until the protection response was near $6 \mu$ s when using optocouplers instead of the CMOS isolated LED emulator Si8710 from Silicon labs and this was an important step in reducing the overall inevitable delay of the proposed overcurrent protection.

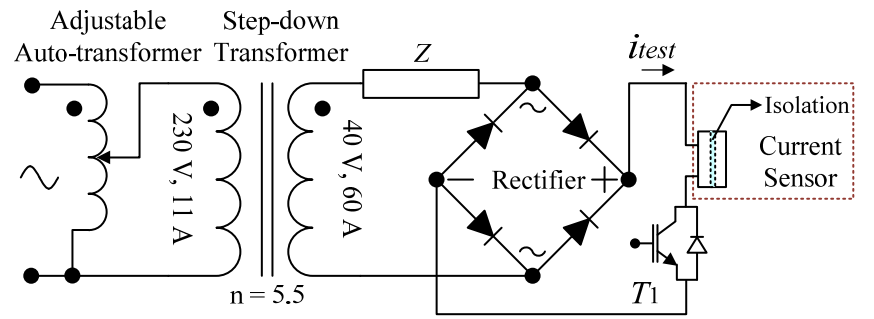

Fig. 7. Schematic of the overcurrent circuit implemented in the workbench for testing the overcurrent protection. 


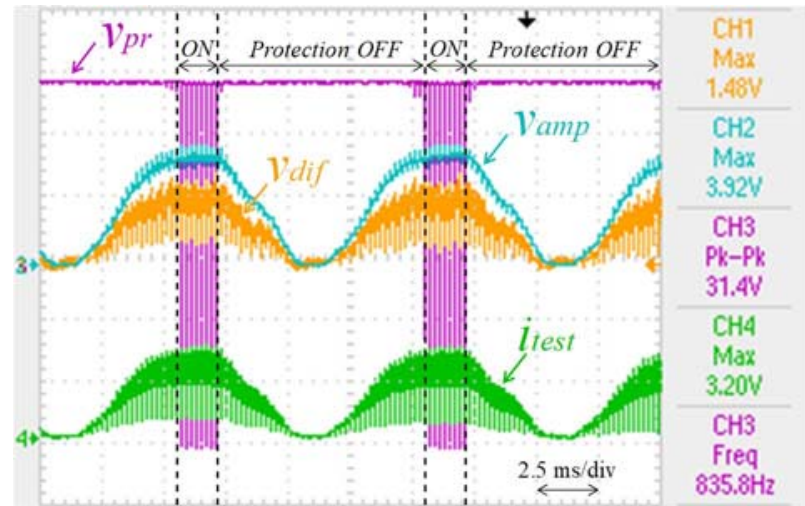

Fig. 8. Experimental results of the proposed overcurrent protection scheme: Test current $\left(i_{\text {test }}: 20 \mathrm{~A} / \mathrm{div}, 100 \mathrm{mV} \leftrightarrow 1 \mathrm{~A}\right)$; Current protection actuating signal ( $\left.v_{p r}: 5 \mathrm{~V} / \mathrm{div}\right)$; Differential output voltage of the current sensor $\left(v_{\text {dif }}: 1 \mathrm{~V} / \mathrm{div}\right)$; Differential output voltage of the current sensor after using a differential amplifier $\left(v_{a m p}: 2 \mathrm{~V} /\right.$ div $)$.

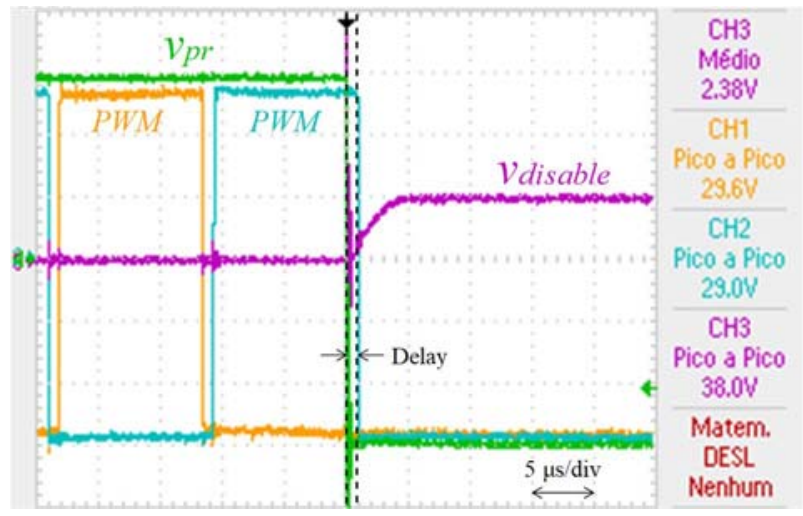

Fig. 9. The response delay from the overcurrent protection actuation until the protection response to disable the $P W M$ pulses. ( $v_{p r}, v_{\text {disable }}, P W M: 5 \mathrm{~V} /$ div $)$.

It is noteworthy to mention that the implemented overcurrent protection is a bidirectional one. By another meaning, it compares the actual current value with its reference value in the positive and the negative cycle of $i_{\text {test }}$ waveform. This is important to avoid fault consequences when the current is flowing through the IGBT or through the antiparallel diode. The target of the proposed overcurrent-protection methodology is to detect harmful currents higher than the peak currents that the semiconductors (IGBT and diodes) can handle. The currents are measured always independently of the direction and the semiconductor device (IGBT or diode) that is carrying such a current. This key feature allows the protection against overcurrent of both, the IGBT and the diode devices. However, the experimental results only show the protection response in the positive half-cycles, since a rectifier bridge is used to avoid the current flow through the diode.

\section{B. Overvoltage Protection Test}

Testing the overvoltage protection of the MMC submodule entails to use a test workbench to validate the behavior of the voltage sensor under test conditions, without damaging the submodule parts. Therefore, in Fig. 10 is presented the overvoltage test workbench which consists of an adjustable autotransformer connected to the input of an uncontrolled rectifier bridge to obtain a $\mathrm{DC}$-voltage on the output terminals.
The overvoltage protection parts are connected to the bridge rectifier output terminals. The resistors $R_{1}, R_{2}$ and $R_{\text {sen }}$ are used as a voltage divider to avoid going beyond the nominal input voltage value of the isolated voltage sensor. The resistor $R_{d i s}$, besides the IGBT $T_{3}$ is used to discharge any extra overvoltage value above the reference signal. It worthy to remember that further attentions have to be considered about the size of the $R_{d i s}$ resistor and its thermal management. For instance, if the resistor is going to be attached to the common heatsink on the submodule, where these aspects have an influence over the submodule volume and reliability. Table. 1 presents the used resistors values of the overvoltage protection.

Fig. 11 shows the experimental results of the overvoltage protection test. The protection has been adjusted to actuate when the submodule voltage exceeds an absolute value higher than $+170 \mathrm{~V}$. The test voltage signal $v_{\text {test }}$ (in orange) has a peak value equal to $174 \mathrm{~V}$ and the overvoltage protection signal $v_{p r}$ (in purple) is actuated when the test voltage exceeds the reference voltage value $(+170 \mathrm{~V})$.

The differential output signal of the voltage sensor after using a differential amplifier $v_{a m p}$ (in cyan) has less noise and a small delay (tens of microseconds) between $v_{t e s t}$ and $v_{a m p}$ signals. This small delay is caused by the voltage sensor delay and the signal conditioning and processing delay. However, the implemented overvoltage protection quickly responds to fault conditions. The signal $v_{\text {ref }}$ (in green) signifies the reference signal in which the protection actuates when $v_{a m p} \geq v_{\text {ref. }}$. The implemented overvoltage protection is a unidirectional one. By another meaning, it only compares the actual voltage value with its reference value in the positive cycles of $v_{\text {test }}$ waveform. This is because the submodule voltage in the MMC is a positive DC one and there is no need to implement a bidirectional voltage protection. A related point to consider in this study is the overcurrent protection delay is extremely lower than the overvoltage protection delay. This is because the voltage in the DC-link capacitors has a slow variation. On the contrary, the current in the IGBT can rapidly change its magnitude and direction. Therefore, the implementation of a robust, fast and effective overcurrent protection is quite a challengeable work.

Table. 1. Resistors values and power ratings of the overvoltage protection.

\begin{tabular}{ccc}
\hline \hline Resistor & Value & Resistor Power Rating at $\mathbf{2 5}^{\circ} \mathbf{C}$ \\
\hline \hline$R_{1}$ & $1 \mathrm{M} \Omega$ & $250 \mathrm{~mW}$ \\
$R_{2}$ & $1 \mathrm{M} \Omega$ & $250 \mathrm{~mW}$ \\
$R_{\text {sen }}$ & $1 \mathrm{k} \Omega$ & $250 \mathrm{~mW}$ \\
$R_{\text {dis }}$ & $5 \mathrm{k} \Omega$ & $14 \mathrm{~W}$ \\
\hline \hline
\end{tabular}

Adjustable

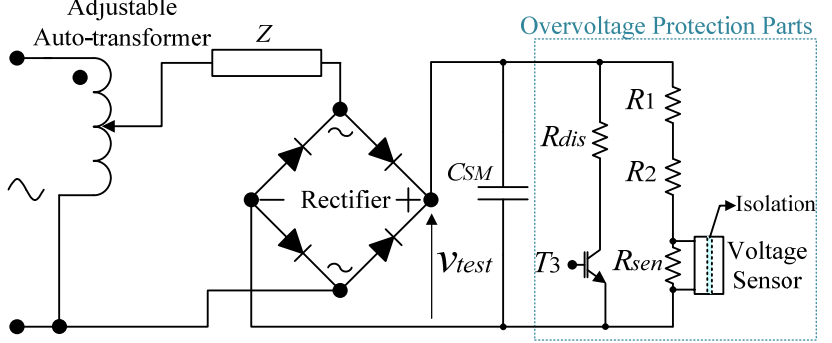

Fig. 10. Schematic of the overvoltage circuit implemented in the workbench for testing the overvoltage protection. 


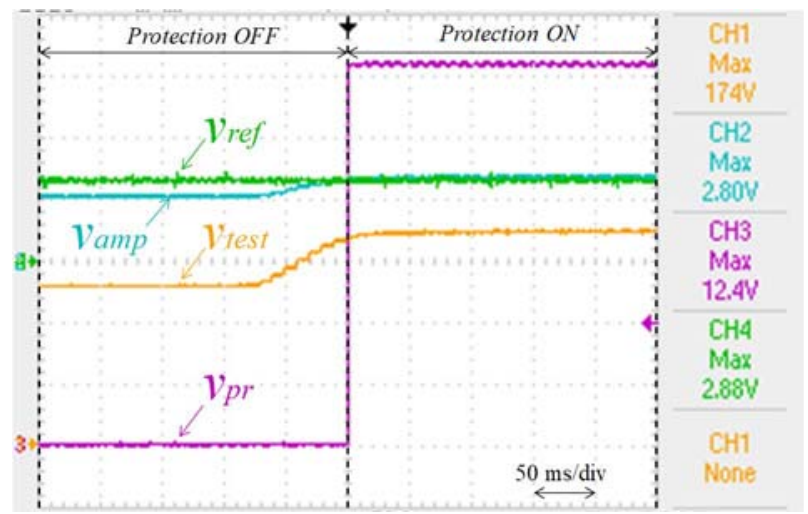

Fig. 11. Schematic Experimental results of the proposed overvoltage protection scheme: Test voltage $\left(v_{\text {test }}: 50 \mathrm{~V} /\right.$ div $)$; Voltage protection actuating signal $\left(v_{p r}: 2 \mathrm{~V} / \mathrm{div}\right)$; Differential output voltage of the voltage sensor after using a differential amplifier $\left(v_{a m p}: 2 \mathrm{~V} / \mathrm{div}\right)$; Reference voltage signal $\left(v_{\text {ref }}: 2\right.$ V/div).

\section{CONCLUSION}

This paper has presented an overview about Modular Multilevel Converters (MMCs) and has explained the importance of the overvoltage and overcurrent protections for an MMC submodule. This study has proposed two protection methods against internal faults for submodules in MMC. In particular, one method covers the protection of $\mathrm{MMC}$ submodules against overcurrent and the second method covers the protection of the submodule against overvoltage. In addition, the study has done a deep and profound explanation about the importance of MMC faults recognition an evaluation. Along the paper, the proposed protection schemes for one submodule and the protection flowchart diagrams have been described. The experimental results for both types of protection have also been presented to validate the proposed protection topologies. Two main experimental tests have been performed by using a special workbench for each practical test, in order to establish operating conditions that are very close to the real ones. Thanks to the innovative sensors technology that grant fast protection responses under different fault situations, hence, the presented results showed a quick protection response when a fault state was detected by the protection system. The proposed protection system represents a new effort to improve the reliability and the stability of MMCs performance.

\section{ACKNOWLEDGMENT}

This work has been supported by national funds through FCT - Fundação para a Ciência e Tecnologia within the Project Scope: UID/CEC/00319/2019. This work has been supported by FCT Project DAIPESEV PTDC/EEI-EEE/30382/2017 and by the ERDF - European Regional Development Fund through the Operational Programme for Competitiveness and Internationalisation - COMPETE 2020 Programme, and by National Funds through the Portuguese funding agency, FCT, within project SAICTPAC/0004/2015 - POCI -01-0145FEDER-016434. Mohamed Tanta was supported by FCT PhD grant with a reference PD/BD/127815/2016.

\section{REFERENCES}

[1] H. Akagi, "Multilevel Converters: Fundamental Circuits and Systems,"Proc. IEEE, vol. 105, no.11, pp.2048-2065, Nov. 2017.

[2] H. Knaak, "Modular multilevel converters and HVDC/FACTS: A success story,"in Proceedings of the 2011 14th European Conference on Power Electronics and Applications, 2011, pp.1-6.

[3] B. Gemmell, J. Dorn, D. Retzmann, and D. Soerangr, "Prospects of multilevel VSC technologies for power transmission," in 2008 IEEE/PES Transmission and Distribution Conference and Exposition, 2008, pp. 1-16.

[4] F. T. Ghetti, A. A. Ferreira, H. A. C. Braga, and P. G. Barbosa, "A study of shunt active power filter based on modular multilevel converter (MMC)," in 2012 10th IEEE/IAS International Conference on Industry Applications, 2012, pp. 1-6.

[5] M. Tanta, V. D. F. Monteiro, J. G. Pinto, A. P. Martins, A. S. Carvalho, and J. L. Afonso, "Efficiency and Cost Estimation for a Static Frequency Converter and a Rail Power Conditioner Based on an Indirect Modular Multilevel Converter in Railways Applications," in ICEE International Conference on Energy and Environment: Bringing Together Engineering and Economics, Porto, Portugal, 2017, pp.313-319.

[6] M. Tanta, J. A. Afonso, A. P. Martins, A. S. Carvalho, and J. L. Afonso, "Rail Power Conditioner Based on Indirect AC/DC/AC Modular Multilevel Converter Using a Three-phase V/V Power Transformer," in Lecture Notes in Engineering and Computer Science: Proceeding of the World Congress on Engineering, 5-7 July 2017, London, UK, pp.289-294.

[7] M. Tanta et al., "Simplified rail power conditioner based on a halfbridge indirect AC/DC/AC Modular Multilevel Converter and a V/V power transformer," in IECON 2017 - 43rd Annual Conference of the IEEE Industrial Electronics Society, 2017, pp.6431-6436.

[8] I. Trintis, S. Munk-Nielsen, and R. Teodorescu, "A new modular multilevel converter with integrated energy storage," in IECON 2011 - 37th Annual Conference of the IEEE Industrial Electronics Society, 2011, pp. 1075-1080.

[9] T. Martel and A. Rufer, "Electric vehicle driving and fast charging system based on configurable modular multilevel converter (CMMC)," in 2013 15th European Conference on Power Electronics and Applications (EPE), 2013, pp. 1-10.

[10] S. Debnath, J. Qin, B. Bahrani, M. Saeedifard, and P. Barbosa, "Operation, Control, and Applications of the Modular Multilevel Converter: A Review," IEEE Trans. Power Electron., vol. 30, no. 1, pp. 37-53, Jan. 2015.

[11] T. Yuan, "Modular multilevel converter: submodule dimensioning, testing method, and topology innovation," PhD thesis, University of Warwick, 2015.

[12] A. A. Aboushady, K. H. Ahmed, and D. Jovcic, "Analysis and Hardware Testing of Cell Capacitor Discharge Currents During DC Faults in Half-Bridge Modular Multilevel Converters," in 11th IET International Conference on AC and DC Power Transmission, 2015, pp. 1-7.

[13] X. Li, Q. Song, W. Liu, H. Rao, S. Xu, and L. Li, "Protection of Nonpermanent Faults on DC Overhead Lines in MMC-Based HVDC Systems," IEEE Trans. Power Deliv., vol. 28, no. 1, pp. 483-490, Jan. 2013.

[14] P. Asimakopoulos, K. Papastergiou, and M. Bongiorno, "Design of a Modular Multilevel Converter As an Active Front-End for a Magnet Supply Application," in 2014 16th European Conference on Power Electronics and Applications, 2014, pp. 1-10.

[15] Y. Li, E. A. Jones, and F. Wang, "Circulating Current Suppressing Control's Impact on Arm Inductance Selection for Modular Multilevel Converter," IEEE J. Emerg. Sel. Top. Power Electron., vol. 5, no. 1, pp. 182-188, Mar. 2017. 